\title{
Application of ZigBee sensor network to data acquisition and monitoring
}

\author{
Mitsugu Terada \\ Department of Applied Physics, Faculty of Science, Fukuoka University, 8-19-1 Nanakuma, Jonan-ku, Fukuoka City, Fukuoka, \\ 814-0180, Japan,mterada@fukuoka-u.ac.jp
}

\begin{abstract}
A ZigBee sensor network for data acquisition and monitoring is presented in this paper. It is configured using a commercially available ZigBee solution. A ZigBee module is connected via a USB interface to a Microsoft Windows PC, which works as a base station in the sensor network. Data collected by remote devices are sent to the base station PC, which is set as a data sink. Each remote device is built of a commercially available ZigBee module product and a sensor. The sensor is a thermocouple connected to a cold junction compensator amplifier. The signal from the amplifier is input to an AD converter port on the ZigBee module. Temperature data are transmitted according to the ZigBee protocol from the remote device to the data sink PC. The data sampling rate is one sampling per second; the highest possible rate is four samplings per second. The data are recorded in the hexadecimal number format by device control software, and the data file is stored in text format on the data sink PC. Time-dependent data changes can be monitored using the macro function of spreadsheet software. The system is considered a useful tool in the field of education, based on the results of trial use for measurement in an undergraduate laboratory class at a university.
\end{abstract}

Keywords: Sensor network, ZigBee protocol, temperature monitoring, automated data display, mechanical equivalent of heat

\section{INTRODUCTION}

$\mathrm{T}$ HE WIRELESS sensor network is expected to be a key technology for several applications such as home automation [1], building control [2], energy saving [3] and automobile monitoring [4]. Several results of investigations of sensor network applications have been reported [5]. Development kit products, in which a temperature sensor, optical sensor, push button switch and beeper are assembled on a circuit board, are provided by ZigBee module vendors for applications such as room lighting and air conditioning controllers. ZigBee sensor networks are potentially suitable for many other applications. ZigBee module products have several types of interfaces; they work at low power consumption, and the modules are usually small. The control software for the module is bundled in the product or is available for free download through the Internet. A system in which data from sensors at remote positions are sent to a base station computer can be configured at a relatively low cost.

A ZigBee sensor network for data acquisition and monitoring is described in this paper. Application in an educational setting is considered to be a new type of sensor network application.

\section{ZIGBEE SENSOR NETWORK}

A wireless sensor network has two main functional aspects: measurement and communication. Each sensor device works as a communication node in the network topology. The network consists of sensor devices that communicate with other devices. As the devices communicate wirelessly, no network infrastructure is necessary. A device joins the network by being deployed in the wireless communication range of a neighbouring device.

ZigBee [6] is the name of a standard that specifies the application layer of a wireless personal area network in a small area and a low communication rate. A near-field smallscale network is easily configured using ZigBee modules. On the other hand, as more than sixty-five thousand devices can be managed by a ZigBee network, a large-scale network can also be configured.

IEEE 802.15.4 specifies the physical layer and medium access control layer of wireless personal area networks [7]. The physical layer is the hardware for network communication. The medium access control layer corresponds to the data link layer of the Open System Interconnection (OSI) reference model. It specifies the method of data transmission and data reception between two neighbouring devices.

A device communicates with neighbouring devices via a wireless link. The radio sensitivity can be changed depending on the environment surrounding the devices. Communication between devices is maintained by selecting an interconnection device, and the communication route changes with every data transmission and reception. The network is typically configured in a star, mesh or cluster tree topology. Devices are classified into two types depending on the supported functions. A reduced function device transmits and receives data signals, and a full function device (FFD) interconnects data signals in addition to transmitting and receiving.

A device transmits data to the $\mathrm{PC}$ via a wireless communication link. The PC works as a base station that controls and manages the network. If a remote device is placed out of the radio-effective area of the PC, its data signal is interconnected by other devices and reaches the PC. A remote device can communicate with the $\mathrm{PC}$ through cooperation of other devices.

\section{TEMPERATURE MONITORING SYSTEM}

A ZigBee network is constructed with ETRX2 devices from Telegesis Limited. One ETRX2 device is connected via a USB interface to a Microsoft Windows PC that works as a base station. The ETRX2 device is set as a data sink, so data from the remote devices are collected by the base station PC.

The following are the basic features of the ETRX2 device used as the ZigBee module: 
Radio frequency: $2.4 \mathrm{GHz}$ band

Data rate: $250 \mathrm{kbit} / \mathrm{s}$

Number of channels: 16 (802.15.4 Channels 11 to 26)

$\checkmark$ I/O port: 12 general-purpose I/O ports and 2 analogue inputs

Typical medium distances: 100-300 metres

Fig.1 shows a remote device that is made for temperature measurement with an ETRX2 device. A thermocouple is connected to a cold junction compensator amplifier. The signal from the amplifier is input to an $\mathrm{AD}$ converter port on the ETRX2 device. As this module works as an FFD, it transmits temperature data and interconnects signals from other measurement devices. A J-type thermocouple is employed for the temperature sensor. The output sensitivity from the amplifier is $10 \mathrm{mV}$ for a temperature of one degree Celsius.

Temperature data are transmitted according to the ZigBee protocol from the remote ETRX2 device to the data sink PC. The data sampling rate is one sampling per second in this application. It can be raised to four samplings per second.

The data are recorded in the hexadecimal number format by Telegesis Terminal software, and a data file is stored in text format on the data sink PC.

Data from remote devices that are successively sent to the data sink PC are recorded in a comma-separated value file. Fig. 2 shows the format of the data. Each line contains four data items. The first item, which is next to the text 'SDATA:', is the ID number of the ZigBee module. The second is the status of 12 digital input/output ports, expressed in four hexadecimal numbers. As one hexadecimal number corresponds to four binary numbers, three out of the four hexadecimal numbers are 12-bit data of digital input/output ports. The third and fourth items are the values of analogue-todigital converters, and correspond to port\#1 and port\#2 of the converters. In this case, the output voltage of the cold junction compensator amplifier is applied to port\#1. The hexadecimal number ' $00 \mathrm{C} 1$ ' is equivalent to the decimal number ' 193 '. It shows that the voltage magnitude applied to port\#1 of the analogue-to-digital converter is $193 \mathrm{mV}$. The temperature is calculated to be 19.3 degree Celsius with a coefficient of 0.1 degree/mV.

As the data sampling rate is one sample/second, the timedependent data change is graphically shown on the PC screen by using the macro function of spreadsheet software. A semireal-time data change is displayed.

\section{Measurement In tRial EXPERIMENT}

A trial measurement was made using the ZigBee measurement system. The aim of this experiment was to confirm that data measured with the ZigBee measurement system are consistent with data measured with the current procedure. The afore-mentioned temperature measurement device is inserted into the present experimental apparatus for mechanical equivalent of heat measurement.

The apparatus used in the experiment are a water calorimeter with an electrical heater element and stirrer, a mercury thermometer, an electrical power supply, an ampere meter and a voltage meter. Fig. 3 shows a schematic of the experimental apparatus for measuring the mechanical equivalent of heat.

All operations are done manually by students in the laboratory classes for a science course at Fukuoka University. Distilled water contained in the copper vessel of the water calorimeter is heated by electrical power supplied to the heater. The value of the mechanical equivalent of heat is calculated from the values of the voltage and current and the difference between the temperatures before and after supplying electrical power to the heater.



Fig.1 Temperature measurement device

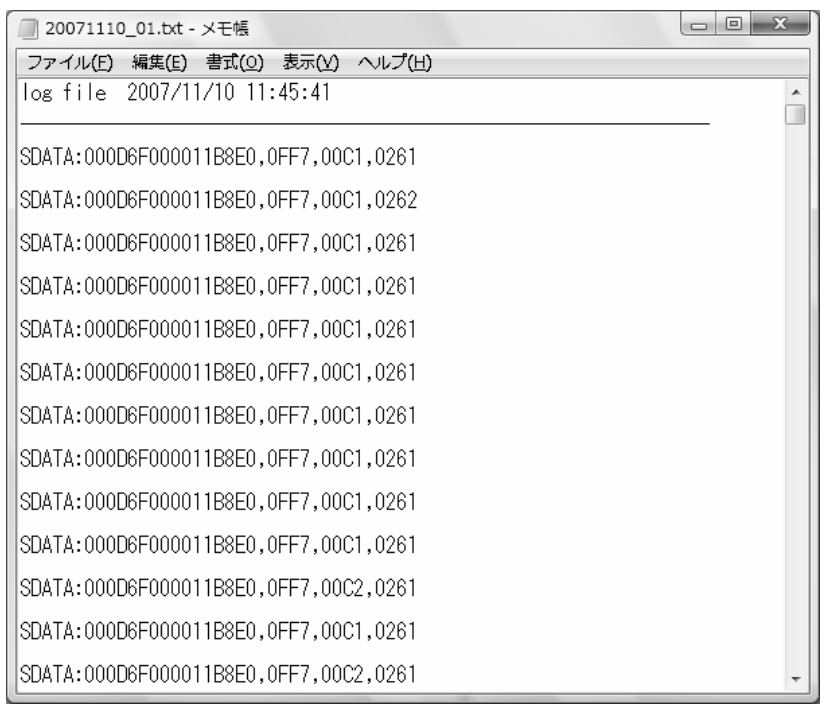

Fig.2 Format of data file

Fig.4 shows the components of the water calorimeter. A thermometer, thermocouple, heater element and stirrer enter the distilled water in the copper container. The water heated by the heater element is stirred in the copper container to keep the water temperature in the container uniform. The water temperature is measured by a thermocouple in the ZigBee measurement system and by a mercury thermometer in the 
manual experimental method. It is important that the water in the copper container is stirred sufficiently and continuously.

The apparatus are left for five minutes to stabilise the temperature of the water in the copper container. The water is heated for five minutes by providing electrical current to the heater. After the heater current is turned off, the apparatus are again left for five minutes to stabilise the water temperature. One experimental period is fifteen minutes.

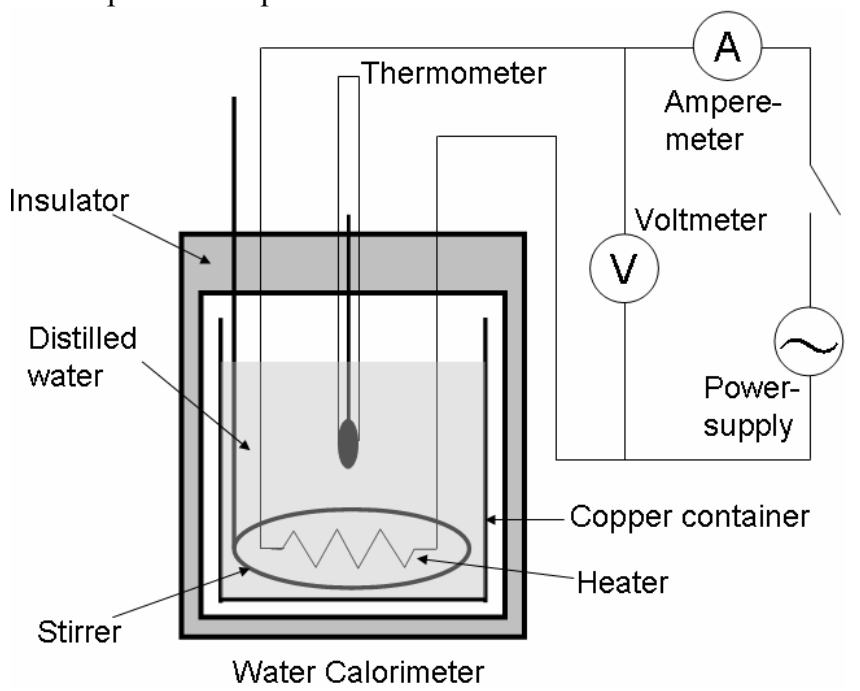

Fig.3 Experimental apparatus

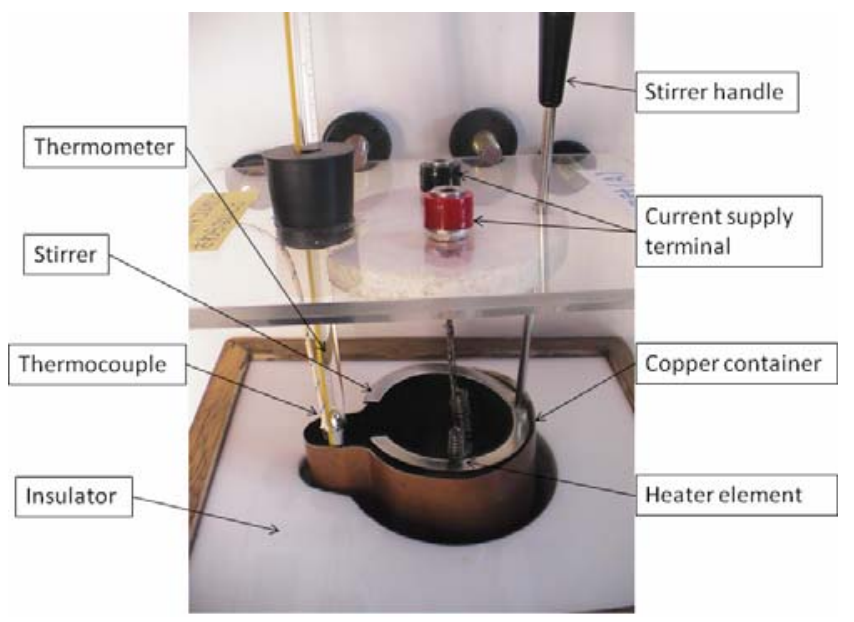

Fig.4 Components of the water calorimeter by

The value of mechanical equivalent of heat $J$ is calculated

$$
J=\frac{V I t}{c(m+w)\left(\theta_{2}-\theta_{1}\right)},
$$

where $I$ is the heater current, $V$ is the voltage between the heater terminals, $t$ is the time for which the water is heated, $c$ is the specific heat of water, $m$ is the mass of the distilled water, $w$ is the water equivalent of the copper container, stirrer and thermometer, and $\theta_{2}-\theta_{1}$ is the difference between the water's temperature before and after heating.
Three measurement operations were made for three stirring conditions: 1) continuous stirring for 15 minutes, one stirring stroke (moving stirrer rod up and down) per second, 2) no stirring at all for 15 minutes and 3) five stirring strokes per five seconds, every 30 seconds for 15 minutes.
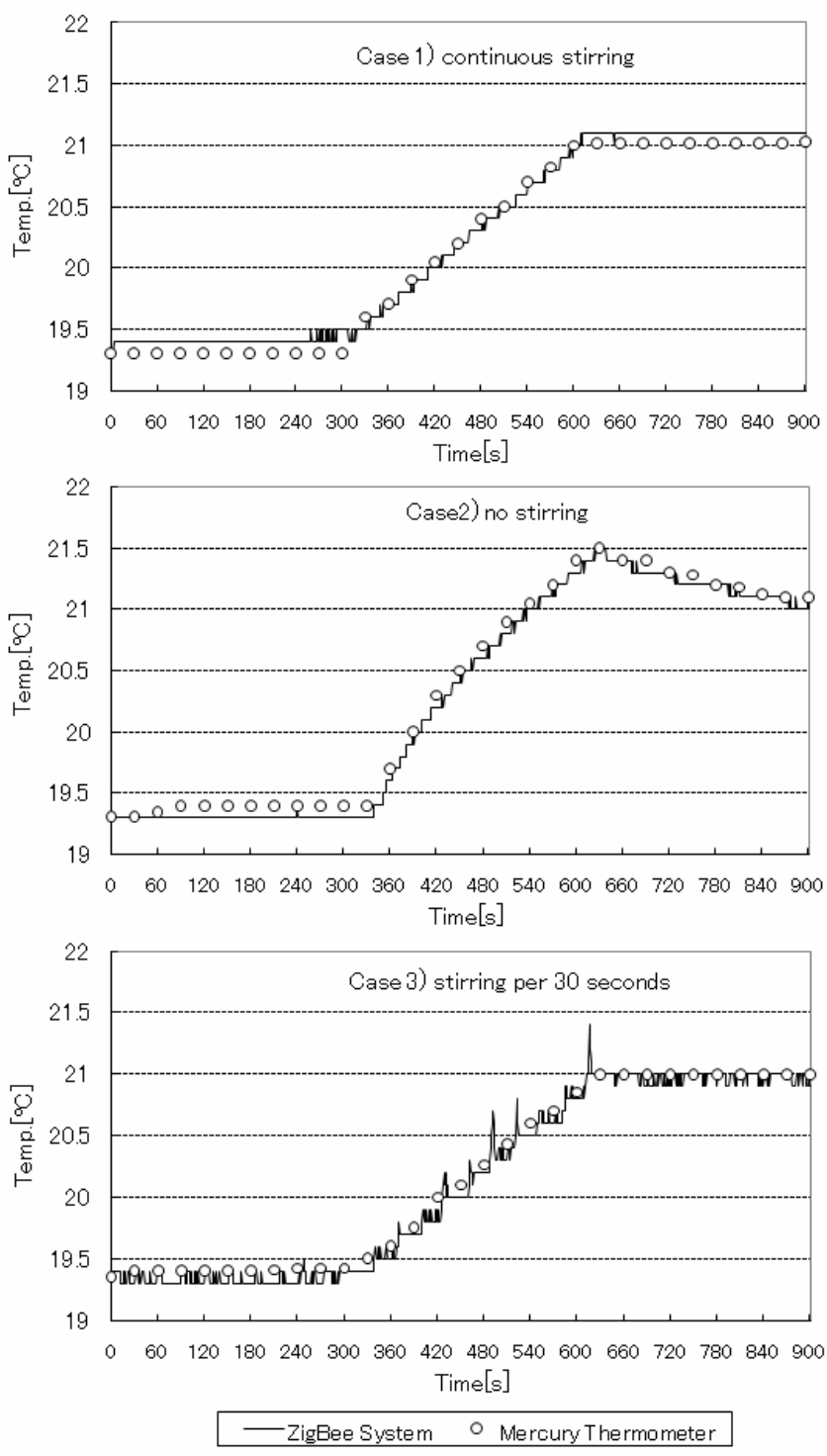

Fig.5 Time dependence of the water temperature for three cases of 1), 2) and 3) where the current fed to the heater is $1.5 \mathrm{~A}$.

Fig.5 shows the time dependence of water temperature change for the three cases and two types of temperature measurement methods. The data taken by the ZigBee system agree with those taken by the mercury thermometer. The ZigBee temperature measurement system is considered suitable for temperature monitoring. The ZigBee system responds more quickly owing to the small thermal capacity of the thermocouple.

Case 1) is considered to be the measurement of uniform water temperature in the container. During the heating period, the water temperature was raised at a constant rate with time. The water temperature remained almost constant in the period after heating. 
In case 2), heat transfers as a convective flow in the water. The rate of water temperature increase was low at the beginning of the heating period. The temperature then increased at a high rate, after which it dropped. The temperature gradually decreased during the period after the heating ended.

Case 3) is an intermediate case. A rapid temperature change occurred after the stirring began. It was not detected by the mercury thermometer. After each instance of stirring, the measured value of the water temperature jumped up and dropped quickly. Later, it stayed almost constant.

The results, temperatures $\theta_{1}$ and $\theta_{2}$, and the value of mechanical equivalent of heat $J$ are shown in Table 1 . The values of $J$ are calculated for $\theta_{1}$ and $\theta_{2}$, which are average temperature values between $t=0 \mathrm{~s}$ and $300 \mathrm{~s}$ and between $t=600 \mathrm{~s}$ and $900 \mathrm{~s}$, respectively. The value of $J$ in Table 1 for case 1, $4.19 \mathrm{~J} / \mathrm{cal}$, agrees well with the defined value of the mechanical equivalent of heat, $4.18605 \mathrm{~J} / \mathrm{cal}$.

Table 1

Temperature data and calculated value of mechanical equivalent of heat for Cases 1), 2) and 3), where $V=2.50 \mathrm{~V}, I=1.50 \mathrm{~A}, t=600 \mathrm{~s}$, $c=1.00 \mathrm{cal} / \mathrm{g}, m=150 \mathrm{~g}$ and $w=8.80 \mathrm{~g}$.

\begin{tabular}{cccc}
\hline \hline & $\theta_{1}\left[{ }^{\circ} \mathrm{C}\right]$ & $\theta_{2}\left[{ }^{\circ} \mathrm{C}\right]$ & $J[\mathrm{~J} /$ cal $]$ \\
\hline Case 1) & 19.40 & 21.10 & 4.19 \\
Case 2) & 19.30 & 21.23 & 3.67 \\
Case 3) & 19.33 & 20.97 & 4.33 \\
\hline \hline
\end{tabular}

Laboratory classes are usually included in the freshman and sophomore courses at science and technology departments of universities. It is difficult for some students to conduct experimental operations because of their lack of experience in the course of high school education. It is necessary to set up a support system for students to conduct their experiments properly and obtain correct results.

It is valuable for the teaching staff (instructors) to discuss the experimental results with students in a laboratory class. As the instructors cover four or five experimental subjects in parallel, they rarely check all students' experimental processes. Four groups of two students each work on an experimental subject, and 16 to 20 subjects are open during a class period (180 minutes). An instructor has to manage 32 to 40 students' experimental operations.

It is worthwhile for the instructors to check ongoing experimental data. The ZigBee data acquisition system is suitable for monitoring the students' experimental processes. Owing to restricted space in existing laboratory facilities and frequent changes in the layout of experimental equipment, the
ZigBee-based data acquisition system is suitable for this situation. A wireless network configuration and small devices are highly preferable for a laboratory data monitoring system.

Students' incorrect operations are found by instructors during the experiment by reviewing the data reported by the monitoring system. The system helps instructors to support students and to instruct students for reasonable results. It is considered a unique application of sensor networks in the field of education.

\section{CONCLUSION}

A temperature measurement system made of sensor devices and ZigBee sensor network modules is applied to automated measurement for laboratory classes at a university. The system is suitable for use in studying the concept of mechanical equivalent of heat in a physics laboratory. Wireless networking and small modules are important points that make this approach suitable in existing laboratory facilities.

\section{REFERENCES}

[1] ZigBee Alliance (2007). ZigBee Home Automation Public Applicataion Profile. Retrieved from http ://www.zigbee.org/Products/TechnicalDocuments Download/tabid/237/Default.aspx

[2] Yu, Y., Prasanna, V.K., Krishnamachari, B. (2006). Information Processing and Routing in Wireless Sensor Networks. World Scientific Publishing Co.

[3] ZigBee Alliance (2007). The Choice for Energy Management and Efficiency. ZigBee White Paper. Retrieved from http://www.zigbee.org/Products/ TechnicalDocumentsDownload/tabid/237/Default.aspx

[4] Tavares, J., Velez, F.J., Ferro, J.M. (2008). Application of wireless sensor networks to automobiles. Measurement Science Review, 8, 65-70.

[5] Wittenburg, G., Terflosth, K., Villafuerte, F.L., Naumowicz, T., Ritter, H., Schiller, J. (2007). Fence monitoring - experimental evaluation of a use case for wireless sensor networks. In Wireless Sensor Network. Lecture Notes in Computer Science. Berlin: Springer, 163-178.

[6] ZigBee Alliance (2006). ZigBee Specification. Retrieved from http://www.zigbee.org/Products/ TechnicalDocumentsDownload/tabid/237/Default.aspx

[7] IEEE Computer Society (2003). IEEE802.15.4 Wireless Medium Access Control (MAC) and Physical Layer (PHY) Specifications for Low-Rate Wireless Personal Area Networks (LR-WPANS). IEEE specification. 